\title{
Genome-wide DNA methylation analysis revealed stable DNA methylation status during decidualization in human endometrial stromal cells
}

Ryo Maekawa, Isao Tamura, Masahiro Shinagawa, Yumiko Mihara, Shun Sato, Maki Okada, Toshiaki Taketani, Hiroshi Tamura and Norihiro Sugino* (D)

\begin{abstract}
Background: During decidualization in endometrial stromal cells (ESCS), expressions of a number of genes and epigenetic modifications of histones are altered. However, there is little information about whether DNA methylation, which is another epigenetic mechanism, also changes during decidualization. Here, we examined the genome-wide DNA methylation profiles in ESCs during decidualization and their associations with the changes of gene expressions and histone modifications.

Results: ESCs were incubated with estradiol and medroxyprogesterone acetate for 14 days to induce decidualization. The genome-wide DNA methylation profiles were compared between the non-decidualized ESCs and the decidualized ESCs. Of 482,005 CpGs, only 23 CpGs (0.0048\%) showed different DNA methylation statuses. The DNA methylation statuses of the differentially expressed genes and the regions with different histone modifications (H3K4 tri-methylation and H3K27 acetylation) were also compared between the ESCs. In the upregulated and downregulated genes in decidualized ESCs, DNA methylation statuses around the promoter region of the genes did not significantly differ between the ESCs. In the regions with different histone modification, DNA methylation statuses did not differ between the ESCs. The differentially expressed genes and the differential histone modification regions were hypomethylated.

Conclusions: Culturing ESCs with estrogen/progesterone did not distort the physiological pattern of DNA methylation, although mRNA expression and histone modifications were dynamically altered. A genome-wide DNA methylation analysis revealed stable DNA methylation statuses during decidualization in human endometrial stromal cells. DNA hypomethylation is maintained for the variable changes of histone modifications and gene expression during decidualization.
\end{abstract}

Keywords: DNA methylation, Decidualization, Endometrial stromal cells, RNA expression, And histone modification

\section{Background}

Human endometrial stromal cells (ESCs) show cyclic changes during the menstrual cycle, which is regulated by estrogen and progesterone. Especially, the process in which fibroblastoid stromal cells of the estrogen-primed endometrium shift into decidual cells by progesterone is called decidualization. Decidualization is crucial for embryo implantation and maintenance of pregnancy $[1,2]$.

\footnotetext{
* Correspondence: sugino@yamaguchi-u.ac.jp

Department of Obstetrics and Gynecology, Yamaguchi University Graduate School of Medicine, Minami Kogushi 1-1-1, Ube 755-8505, Japan
}

The expressions of a number of genes change during decidualization, suggesting that decidualization is accompanied by dramatic changes of cell functions. Gene expression includes a change of chromatin structure, which can be regulated by epigenetic mechanisms such as DNA methylation and histone modifications [3, 4]. Recently, we found that active marks of histone modifications such as histone H3K4 tri-methylation (H3K4me3) and histone H3K27 acetylation (H3K27ac) dramatically changed in ESCs during decidualization, and these histone modifications were associated with the changes of gene expression [5].

(c) The Author(s). 2019 Open Access This article is distributed under the terms of the Creative Commons Attribution 4.0 International License (http://creativecommons.org/licenses/by/4.0/), which permits unrestricted use, distribution, and reproduction in any medium, provided you give appropriate credit to the original author(s) and the source, provide a link to the Creative Commons license, and indicate if changes were made. The Creative Commons Public Domain Dedication waiver (http://creativecommons.org/publicdomain/zero/1.0/) applies to the data made available in this article, unless otherwise stated. 
DNA methylation is a well-characterized epigenetic mark. DNA methylation, which occurs at CpG sites, interrupts the recognition and binding of transcription factors [6-10], recruits methyl CpG-binding proteins that interact with transcription repressors [7, 11], and induces chromatin condensation via histone modification changes $[7,12]$. DNA hypermethylation at promoter regions is associated with gene silencing, while DNA hypomethylation is associated with gene activation [7]. In most of the genes including housekeeping genes, the promoter regions are DNA hypomethylated in all types of cells/tissues. On the other hand, there are genes whose DNA methylation statuses are specific to each cell/tissue and have important roles in determining the cell/tissue-specific gene expression profiles [7, 13, 14]. To better understand decidualization, it is of interest to know 1) whether the DNA methylation profiles change and contribute to the changes of gene expression profiles in ESCs during decidualization and 2) how two major epigenetic marks, DNA methylation and histone modifications, are intertwined with each other during decidualization.

In the present study, we compared the genome-wide DNA methylation profiles between non-decidualized ESCs and decidualized ESCs. We next compared the DNA methylation statuses of the genes whose mRNA expression profiles differed between the non-decidualized ESCs and the decidualized ESCs. Since histone modifications are known to interact with DNA methylation [15-18], we compared the DNA methylation statuses of the regions in which histone modification (H3K4me3 and H3K27ac) statuses were different between the non-decidualized ESCs and the decidualized ESCs.

\section{Results}

\section{Genome-wide DNA methylation profiles of ESCs}

To assess the overall patterns of DNA methylation, we examined the DNA methylation levels of 482,005 CpGs in the ESCs. The average DNA methylation levels (beta-values) of the CpGs in the non-decidualized and decidualized ESCs were $0.468 \pm 0.364$ and $0.465 \pm 0.366$, respectively. As shown in Fig. 1a, in the non-decidualized and decidualized ESCs, the majority of CpGs were hypomethylated (beta-value $<0.2 ; 38.62$ and $38.96 \%$, respectively) or hypermethylated (beta-value $>0.7 ; 40.10$ and $39.98 \%$, respectively), and the remaining CpGs had intermediate levels of DNA methylation $(0.2 \leq$ beta-value $\leq 0.7$; 21.27 and $21.05 \%$, respectively). There was no significant difference in the distribution patterns of the methylated CpGs between the decidualized and non-decidualized ESCs.

To assess the number of the CpGs that were differentially methylated between the non-decidualized and decidualized ESCs, the beta-values of all CpGs in both
ESC types were plotted in a scatter plot (Fig. 1b). The scatter plot showed a linear distribution pattern, indicating that there were few differences in the DNA methylation levels between the two groups. In fact, when the cut-off of the beta-value is more than 0.3, which is generally accepted, only 23 CpGs were differentially methylated (Table 1).

We compared the DNA methylation profiles based on the gene structures between the ESCs. In the present study, we obtained the DNA methylation statuses of 482,005 CpGs among 41,204 genes. The CpGs were grouped into three regions: upstream region (from $1500 \mathrm{bp}$ to transcription start site), gene body region (from transcription start site to transcription end site) and downstream region (from transcription end site to $+1000 \mathrm{bp}$ ) of the genes. These regions included 253,082, 564,325 and 22,012 CpGs, respectively. As shown in Fig. 1c, the DNA methylation patterns of the three regions were very similar between the two ESC types, and the DNA methylation status was hypomethylated in the promoter region ( $-500 \mathrm{bp}$ to transcription start sites) in the both ESCs.

\section{DNA methylation profiles of differentially expressed genes}

We examined the DNA methylation statuses around the transcription start sites of differentially expressed genes between the non-decidualized and decidualized ESCs. We obtained 2419 up-regulated and 2276 down-regulated genes in the decidualized ESCs compared with the non-decidualized ESCs from our previous study [5] (Additional file 1: Table $\mathrm{S} 1)$. In these genes, the DNA methylation statuses from 1500 to $4000 \mathrm{bp}$ were compared between the ESCs. Among the up-regulated 2419 genes, the regions around the transcription start sites (from -300 to $+700 \mathrm{bp}$ ) were DNA hypomethylated compared to the other regions in both the non-decidualized ESCs (beta-value: $0.096 \pm 0.170$ ) and the decidualized ESCs (beta-value: $0.093 \pm 0.170$ ) (Fig. 2a). Similarly, among the down-regulated 2276 genes, the regions around the transcription start sites (from -300 to $+700 \mathrm{bp}$ ) were hypomethylated in the both ESC types (beta-values: $0.104 \pm 0.183$ and $0.101 \pm 0.183$, respectively) (Fig. 2b). These results indicate that there was no significant difference in the DNA methylation levels around the transcription start sites of the differentially expressed genes between the two ESC types.

Our finding that the DNA hypomethylation around the transcription start sites of the differentially expressed genes is concomitant with the fact that DNA hypomethylations of the promoter regions are associated with gene expression $[18,19]$. Therefore, we hypothesized that the genes that were not expressed in either type of ESC were hypermethylated. Therefore, we examined the DNA methylation statuses of these genes (those with FPKM <0.01). The 

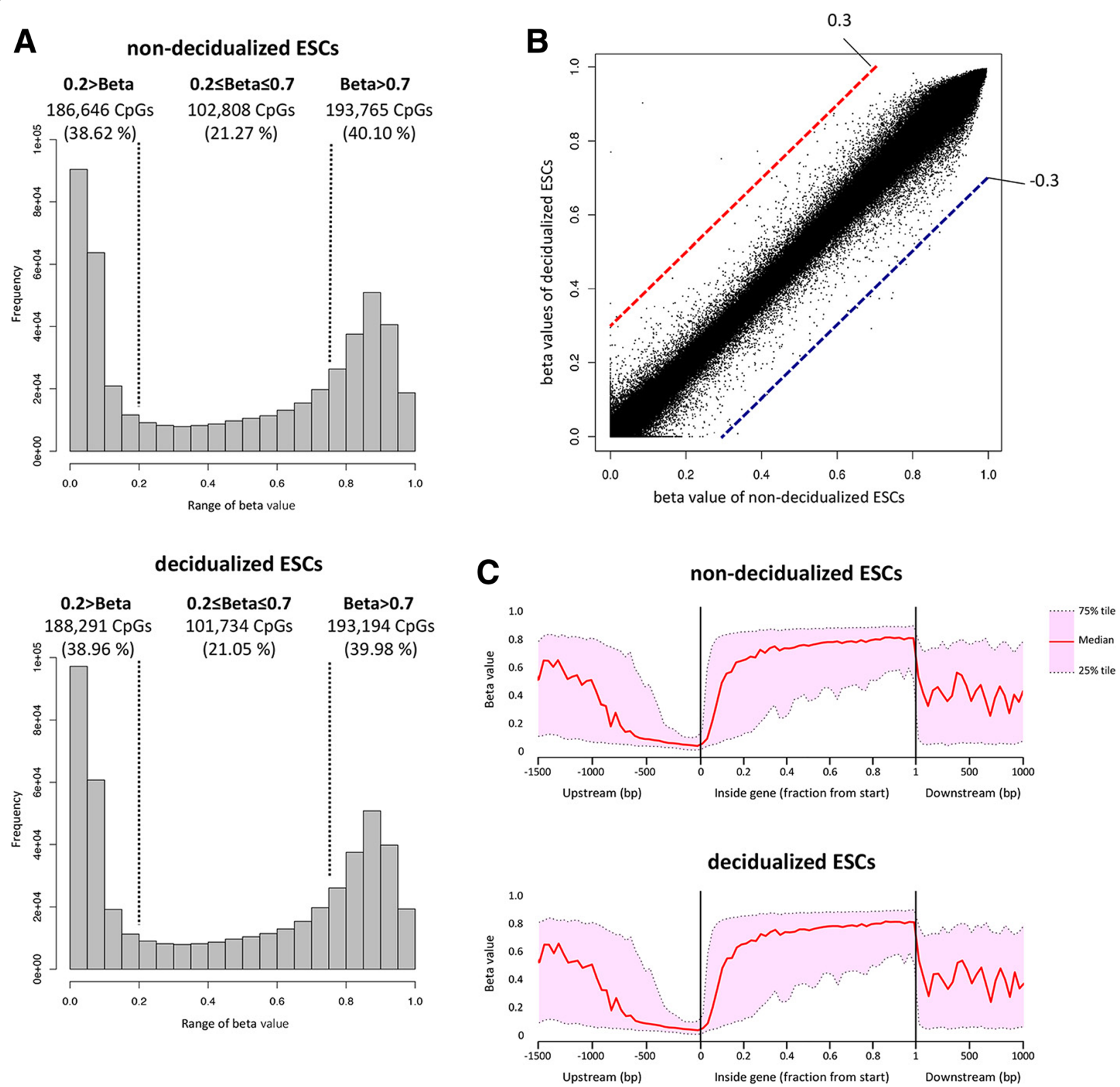

Fig. 1 Genome-wide DNA methylation profiles of the non-decidualized and decidualized ESCs. a: Histograms of the DNA methylation levels (betavalues) of 482,005 CpGs in the non-decidualized and decidualized ESCs. In each range of beta-values $(0.2>, 0.2$ to 0.7 , and $0.7<)$, the frequencies and the ratios of the methylated CpGs to all CpGs are shown. $\mathbf{b}$ : Scatterplot of the beta-values in the non-decidualized and decidualized ESCs. The CpGs are plotted according to the beta-values of the non-decidualized (horizontal axis) and decidualized ESCs (vertical axis). Dashed lines show minimum beta-value changes of 0.30. Red dashed lines indicate the cut-off beta-values for the CpCs which have increased beta-values during decidualization while blue dashed lines indicate the cut-off values for the CpGs which have decreased beta-values. c: DNA methylation level vs. gene position in the non-decidualized (top) and decidualized ESCs (bottom). The DNA methylation profiles of upstream region (from - 1500 bp to transcription start site), gene body region (from transcription start site to transcription end site) and downstream region (from transcription end site to $+1000 \mathrm{bp}$ ) of the genes are shown. In the gene body region, the distance from transcription start site to transcription end site was rescaled into the range of [0-1] as previously reported [18]. Median (50\%), 25 and 75\% tile beta-values are plotted in the non-decidualized and decidualized ESCs (c). Vertical and horizontal lines show beta-values the position relative to the transcription start site

beta-values of the genes without expression in both ESCs were $0.349 \pm 0.339$ in non-decidualized ESCs and $0.347 \pm 0.341$ in decidualized ESCs (Fig. 2c). The genes that were not expressed in either type of ESC were more highly methylated than the differentially expressed genes (Fig. 2d). This suggests that DNA hypomethylation is necessary for differential gene expression between the non-decidualized and decidualized ESCs. 
Table 1 The number of the differentially methylated CpGs

\begin{tabular}{lll}
\hline Cut-off of beta value & $0.15<$ & $0.30<$ \\
\hline Total & $582(0.1203 \%)$ & $23(0.0048 \%)$ \\
Hypomethylated CpG sites & 210 & 8 \\
Hypermethylated CpG sites & 372 & 15
\end{tabular}

Human endometrial stromal cells were treated with or without $\mathrm{E}$ and MPA for 14 days. DNA methylation statuses of the non-decidualized and decidualized ESCs were analyzed by Illumina Infinium HumanMethylation450 BeadChip. The number of the differentially methylated CpG sites was calculated when the difference of DNA methylation levels (beta-value) between the two types of ESCs was more than 0.15 and 0.3

\section{Differentially methylated genes and their mRNA expression statuses}

Of 23 differentially DNA methylated CpG sites, 11 CpG sites were associated with 11 genes (Table 2). Of 11 genes, three genes (transcription factor AP-2 alpha, TFAP2A; ubiquitin conjugating enzyme E2 D2, UBE2D2; component of oligomeric Golgi complex 5, COG5) differed in mRNA expression. TFAP2A was more methylated and less expressed in the decidualized ESCs, while UBE2D2 and COG5 were less methylated and more expressed in the decidualized ESCs. The different DNA methylation statuses of UBE2D2 and COG3 were validated by sodium bisulfite sequencing (Additional file 6: Figure S1).

\section{DNA methylation profiles of regions with histone modification change during decidualization}

Because DNA methylation interacts with other epigenetic features such as histone modifications [15-18], we investigated the DNA methylation statuses in regions in which histone modifications were altered by decidualization according to our previous study [5]. We obtained the regions with increased or decreased histone modifications of H3K4me3 (3465 and 2841 regions, respectively) and H3K27ac (13,119 and 829 regions) during decidualization (Additional file 2: Table S2) [5]. In H3K4me3-increased and -decreased regions, the DNA methylation statuses did not differ significantly between the ESCs (Fig. 3a). In the H3K27ac-increased and -decreased regions, the DNA methylation statuses also did not differ significantly between the ESCs (Fig. 3b). These results also indicate that the regions in which histone modification was altered during decidualization showed DNA hypomethylation status in both types of ESC (Fig. $3 \mathrm{a}$ and $\mathrm{b}$ ). This suggests that DNA hypomethylation is critical for changes in histone modification during decidualization. Therefore, we investigated whether the regions that had no histone modification in either type of ESC were DNA hypermethylated. As shown in Fig. 3c, the regions without $\mathrm{H} 3 \mathrm{~K} 4 \mathrm{me} 3$ in both types of ESC were more methylated than the regions with different histone modifications between the ESCs (Fig. 3a). Similarly, the regions without $\mathrm{H} 3 \mathrm{~K} 27 \mathrm{ac}$ in both types of $\mathrm{ESC}$ were more methylated (Fig. 3d) than the regions with different histone modifications between the ESCs (Fig. 3b). There was no significant difference in the DNA methylation statuses between the non-decidualized and decidualized ESCs (Fig. 3c and d).

\section{DNA methylation and histone modification statuses of IGFBP-1 and PRL}

IGFBP-1 and PRL are representative markers for decidualization that are upregulated in decidualization [1]. The DNA methylation statuses of the promoter region of IGFBP-1 and PRL were examined by bisulfite sequencing. The IGFBP-1 promoter region has $27 \mathrm{CpGs}$ between $-501 \mathrm{bp}$ to about $+75 \mathrm{bp}$ and the PRL promoter and enhancer regions have 11 CpGs between - 1966 bp to about $+350 \mathrm{bp}$. In the non-decidualized ESCs, the CpGs in the proximal region of IGFBP-1 promoter, which exist near the transcriptional factor binding sites $(-263$ to $+75 \mathrm{bp})$, were hypomethylated, whereas the CpGs in the distal region ( -501 to $-442 \mathrm{bp}$ ) were methylated (Additional file 7: Figure S2A). The DNA methylation status in the IGFBP-1 promoter region was not altered by decidualization (Additional file 7: Figure S2A). As for PRL, two CpGs (-4 and-360 bp) were hypomethylated and the other CpGs were DNA methylated in the non-decidualized ESCs (Additional file 7: Figure S2B). The DNA methylation status in the $P R L$ promoter region was not altered by decidualization (Additional file 7: Figure S2B). We next investigated whether the histone modification (H3K27ac and H3K4me3) in the promoter region changes during decidualization under the DNA hypomethylation status. As shown in Additional file 7: Figure S2C, the H3K27ac of IGFBP-1 showed an increased modification, while that of $P R L$ didn't show a change during decidualization. The H3K4me3 were not altered by decidualization.

\section{Comparison of the DNA methylation status between ESCs and other cell types}

To investigate whether DNA hypomethylated genes in ESCs with differential expression before and after decidualization exhibit DNA hypermethylation statuses in cell types other than ESCs, we examined the DNA methylation statuses of these genes in publicly available Illumina HumanMethylation450 data of embryonic stem cells, brain, liver, omentum, pancreas, spleen, saliva and leucocyte. We first extracted the genes whose average beta-value from - 300 to +700 bp in the ESCs was $<0.2$ and whose expression were up-regulated or down-regulated during decidualization (Additional file 4: Table S4). As a result, 1764 up-regulated and 1596 down-regulated genes were obtained. In 1764 up-regulated genes, a number of genes (107 to 258 genes) were differentially DNA hypermethylated in other cell types compared to the ESCs ( $\triangle$ beta-values >0.3, Fig. 4a). 


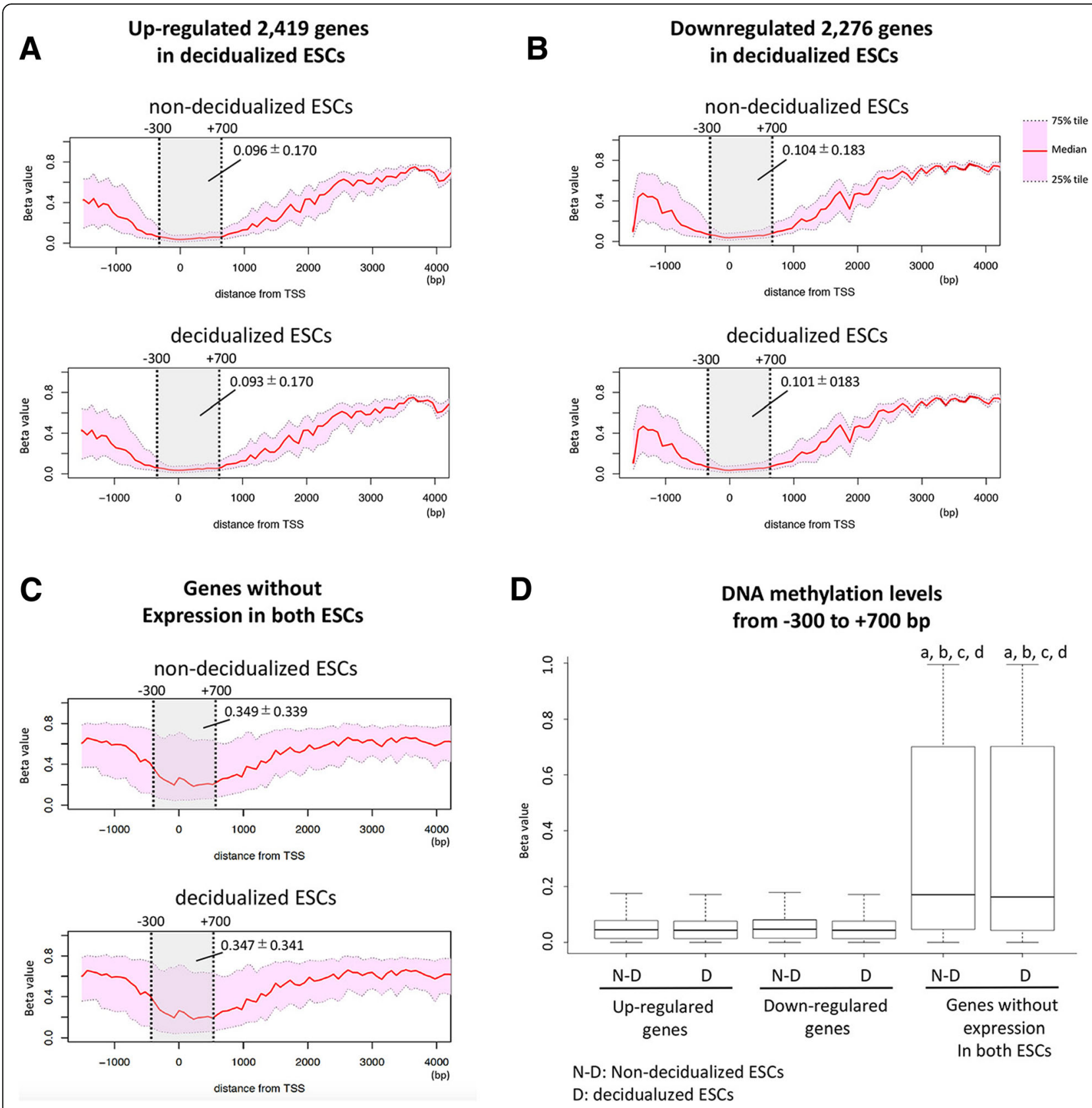

Fig. 2 DNA methylation status of differentially expressed genes between the non-decidualized and decidualized ESCs. a and b: Median (50\%), 25 and $75 \%$ tiles of beta-values of up-regulated 2419 genes (a) and down-regulated 2276 genes (b) are shown in the non-decidualized and decidualized ESCS. Vertical and horizontal lines show beta-values the position relative to the transcription start site (from - 1500 to +4000 bp). c: Median (50\%), 25 and $75 \%$ tiles of beta-values of the genes without expression in both types of ESC (9328 genes) are shown in the non-decidualized and decidualized ESCS. d: DNA methylation levels (mean \pm SD, from -300 to +700 ) of the non-decidualized and decidualized ESCs of up-regulated genes, downregulated genes, and genes without expression in both types of ESC are shown in boxplots. a, $p<0.01$ vs. the non-decidualized ESCs of up-regulated genes; $\mathbf{b}, p<0.01$ vs. the decidualized ESCs of up-regulated genes; $\mathbf{c}, p<0.01$ vs. the non-decidualized ESCs of down-regulated genes; $\mathbf{d}, p<0.01$ vs. the decidualized ESCS of down-regulated genes

Similarly, in 1596 down-regulated genes, a number of genes (95 to 261 genes) were DNA hypermethylated in other cell types (Fig. 4a). We next investigated the genes that are specifically DNA hypomethylated in ESCs. Of the 1764 up-regulated genes, 381 genes were less methylated in the ESCs than in at least one cell type and 63 genes showed ESC-specific DNA hypomethylation (Fig. 4b and Additional file 4: Table S4). In 1596 down-regulated genes, 322 genes were less methylated in the ESCs than in at least one cell type and 43 genes showed ESC-specific 
Table 2 Eleven differentially methylated genes between non-decidualized and decidualized ESCs and mRNA expression statuses

\begin{tabular}{|c|c|c|c|c|}
\hline \multirow[t]{2}{*}{ Genes (Refseq IDs) } & \multirow{2}{*}{$\begin{array}{l}\text { Gene } \\
\text { symbols }\end{array}$} & \multicolumn{2}{|c|}{ DNA methylation (beta values) } & \multirow{2}{*}{$\begin{array}{l}\text { Expression ratio (Fold change) } \\
\text { decidualized ESCs/non-decidualized ESCs }\end{array}$} \\
\hline & & non-decidualized ESCs & decidualized ESCs & \\
\hline NM_001032280 & TFAP2A & 0.001116211 & 0.7699487 & 0.602183089 \\
\hline NM_001099284 & ZNF239 & 0.3894365 & 0.7715948 & 0.983577659 \\
\hline NM_006897 & $\mathrm{HOXC9}$ & 0 & 0.3599297 & 0.957752628 \\
\hline NR_001317 & HCG4P6 & 0.0165225 & 0.3242724 & 0.935774769 \\
\hline NM_016078 & FAM18B & 0.004547609 & 0.3047889 & 1.023612243 \\
\hline NM_176810 & NLRP13 & 0.5744109 & 0.2743416 & 1 \\
\hline NM_033554 & HLA-DPA1 & 0.6535866 & 0.3476084 & 0.915018217 \\
\hline NM_006557 & DMRT2 & 0.8715751 & 0.5523196 & 1 \\
\hline NM_181733 & COG5 & 0.7118202 & 0.3734171 & 1.801646442 \\
\hline NM_000257 & MYH7 & 0.7474061 & 0.3890077 & 1 \\
\hline NM_181838 & UBE2D2 & 0.7447599 & 0.374194 & 1.885683062 \\
\hline
\end{tabular}
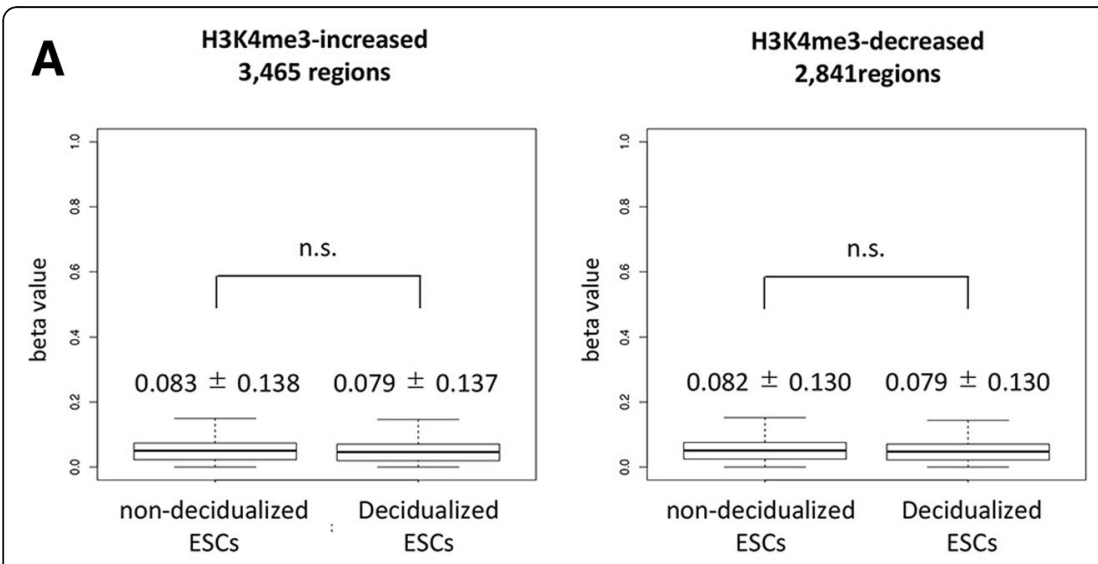

\section{C}

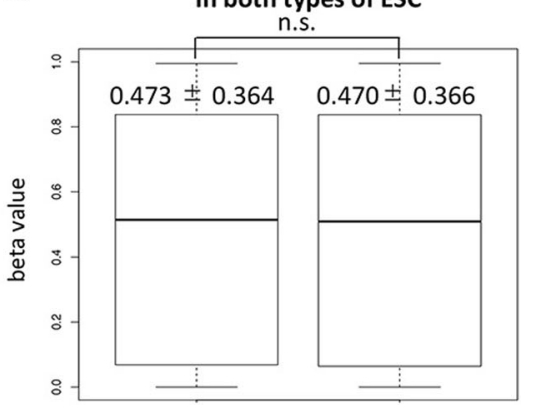

$$
\begin{array}{cc}
\text { non-decidualized } & \text { Decidualized } \\
\text { ESCS } & \text { ESCS }
\end{array}
$$

Degions without H3K27ac in both types of ESC

\section{B \\ H3K27ac-increased 13,119 regions}

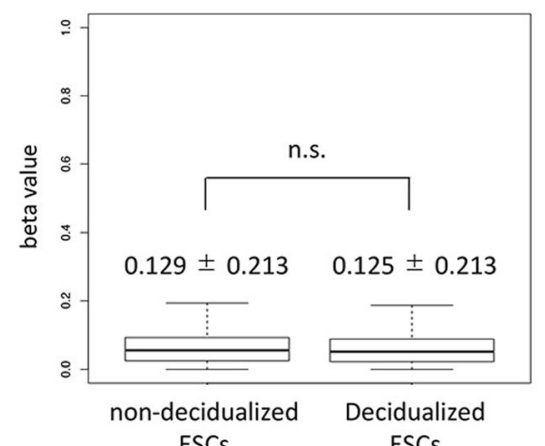

H3K27ac-decreased 829 regions

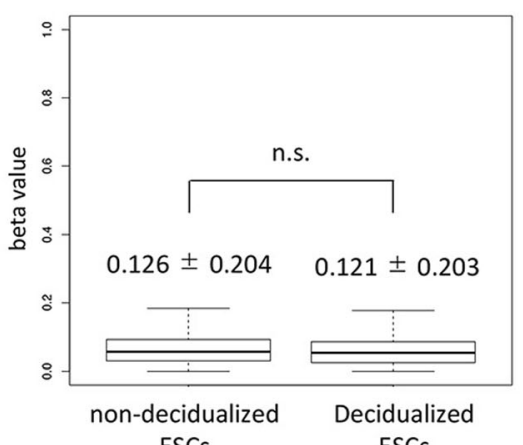
n.s.

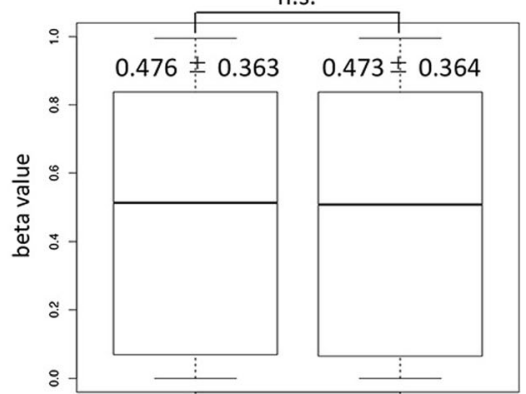

non-decidualized Decidualized ESCS ESCS

Fig. 3 DNA methylation statuses of the differentially histone modified regions between the non-decidualized and decidualized ESCs. a: The betavalues of H3K4me3-increased (3465 regions, left) and H3K4me3-decreased (2841 regions, right) are shown in boxplots. Each mean and SD value is shown above the boxplot. b: The beta-values of H3K27ac-increased (13,119 regions, left) and H3K27ac-decreased (829 regions, right) are shown in boxplots. $\mathbf{c}$ : The beta-values of the regions without H3K4me3 in both types of ESC are shown in boxplots. $\mathbf{d}$ : The beta-values of the regions without H3K27ac in both types of ESC are shown in boxplots. n.S., not significant 
A
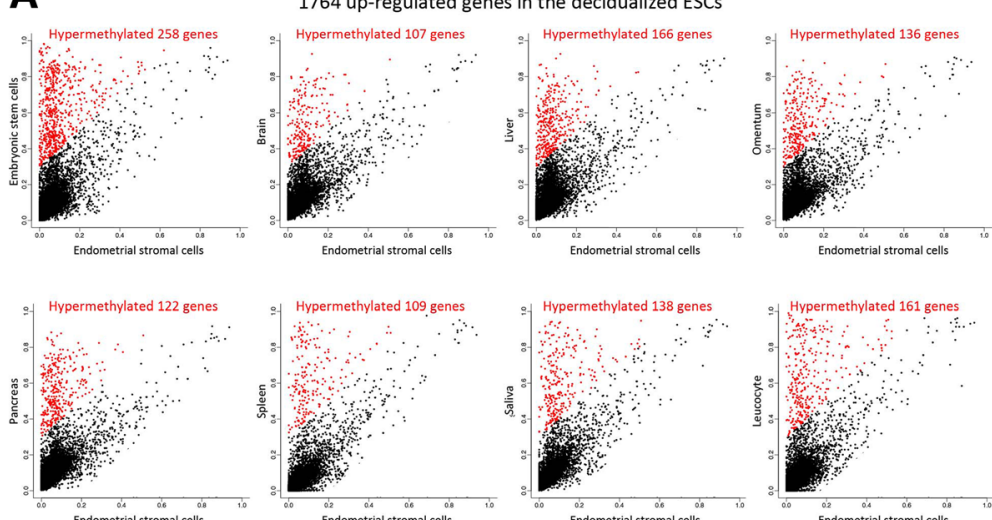

1596 down-regulated genes in the decidualized ESCs
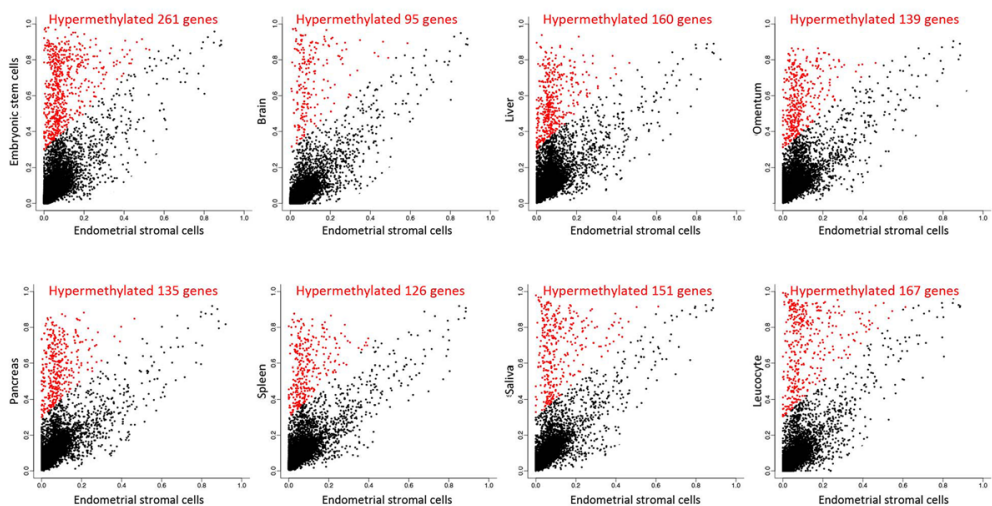

B

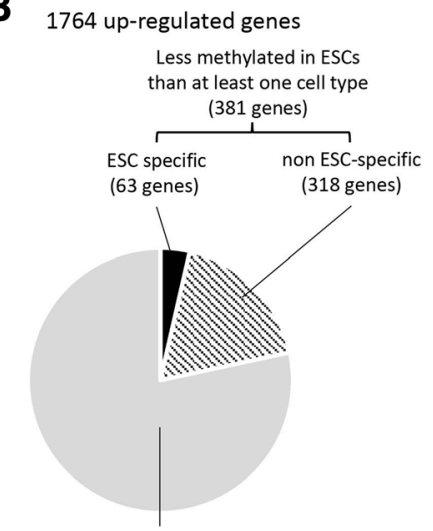

1596 down-regulated genes Less methylated in ESCS than at least one cell type (322 genes)

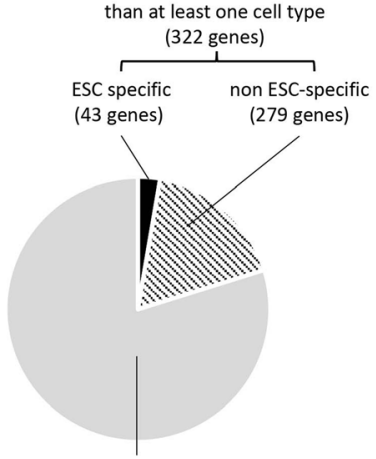

Not differentially methylated genes (1383 genes)

Not differentially methylated genes (1274 genes)

Fig. 4 Comparison of the DNA methylation status between the ESCs and other cell types. a: Scatterplots of the beta-values in the ESCs and other cell types. The DNA methylation statuses of the genes whose average beta-value from -300 to $+700 \mathrm{bp}$ in the ESCs was less than 0.2 and whose expression were increased or decreased more than 1.5-fold during decidualization were compared between the ESCs and other cell types (human embryonic stem cells, brain, liver, omentum, pancreas, spleen, saliva and leukocyte). The average beta-values from -300 to +700 bp are plotted according to the beta-values of the ESCs (horizontal axis) and other cell types (vertical axis). Dashed lines show minimum beta-value changes of 0.30. Red dots indicate the genes that are differentially DNA hypermethylated ( $\Delta$ beta-values $>0.3$ ) genes in other cell types compared to the ESCs. b: Up-regulated and down-regulated genes are classified into 3 groups based on the specificity of the DNA hypomethylation in the ESCs. ESC-specific genes indicate the genes that are less methylated in the ESCs than any other cell types. Non-ESC-specific genes indicate the genes in which one or more cell types show DNA hypomethylation similar to the ESCs 
DNA hypomethylation (Fig. 4b and Additional file 4: Table S4). In $106(63+43)$ of ESC-specific hypomethylated genes, Gene Ontology (GO) analysis showed that these genes included metalloproteinase and collagen genes which compose extracellular matrix and interstitial matrix (Additional file 5: Table S5).

\section{The expression statuses of the ESC-specific hypomethylated genes in other cell types}

We next evaluated the ESC-specific DNA hypomethylation was linked to ESC-specific gene expression. We examined the expression statuses of the ESC-specific hypomethylated and differentially expressed genes during decidualization (63 up-regulated and 43 down-regulated genes) in publicly available RNA sequencing data of embryonic stem cells, brain, liver, pancreas, spleen and saliva. Then the expression levels were compared between the ESCs and other cell types. The ESC-specific hypomethylated and up-regulated genes showed significantly lower expression statuses in brain, liver, pancreas, spleen and saliva than the decidualized ESCs $(p<0.01$, Additional file 9: Figure S4). The ESC-specific hypomethylated and down-regulated genes showed significantly lower expression statuses in other cell types than the non-decidualized ESCs $(p<0.05$, Additional file 9: Figure S4). These results indicate that the differentially expressed genes during decidualization with ESC-specific hypomethylation were kept down-regulated in other cell types.

\section{Discussion}

Genome-wide DNA methylation analysis revealed no remarkable difference in DNA methylation profiles between the non-decidualized and decidualized ESCs. Only $0.0048 \%$ ( $\Delta$ beta-value $>0.3$ ) of the examined CpG sites were differentially methylated between the two types of ESC. DNA methylation is specific to each cell/tissue type [7], and is involved in cell/tissue-specific gene expression $[13,14]$. During cell differentiation, DNA methylation changes occur early in cell lineage commitment, and only a few changes arise during the terminal differentiation [20]. Transcriptional repression of genes by DNA methylation is essentially limited to specific genes that need to be permanently silenced. These observations suggest that once cells are terminally differentiated, the DNA methylation status is stable and does not change easily [21]. Therefore, it is not surprising that DNA methylation did not change in the ESCs during decidualization. Decidualization would be a cellular differentiation of ESCs to another cell type only with gene expression changes.

The DNA methylation statuses around the gene promoter and within the gene body are closely associated with mRNA expression [18, 19]. Most of the 2419 up-regulated and 2276 down-regulated genes didn't show any difference in the DNA methylation statuses between the two types of ESC. This result indicates that DNA methylation is not directly associated with the change of gene expression during decidualization. Notably, the present study showed that the DNA methylation statuses of the promoter regions of the genes with different expression between the two types of ESCs were hypomethylated, while those of the genes that are not expressed in either type of ESC were hypermethylated. This is concomitant with the general finding that DNA hypomethylation around the transcription start sites is associated with gene expression [7]. Furthermore, this finding suggests that the DNA hypomethylation of the gene promoter region underlies the variability of the gene expression during decidualization, and the expression of the genes with DNA hypomethylation at the promoter region is regulated by other mechanisms such as histone modifications and transcription factor binding $[4,22]$.

Our previous study showed that histone modifications were genome-widely altered in the ESCs during decidualization [5]. Since histone modifications interact with DNA methylation each other, we investigated in the present study whether the DNA methylation statuses were also altered in the regions in which histone modifications were altered during decidualization. The results showed that there was no difference in the DNA methylation statuses of those regions between the non-decidualized and decidualized ESCs. This is not surprising because histone modifications are involved in short-term and variable epigenetic regulation, while DNA methylation does not play a role in such fine-tuning of gene expression [23]. Notably, the regions in which histone modifications were altered during decidualization showed DNA hypomethylation statuses compared with the regions without the histone modification changes in the both types of ESC. Active histone modifications under the DNA hypomethylation status induce loosened chromatin structures, which promote the access of transcription factors to the gene promoter regions to induce gene expression [24, 25]. In the present study, we found that DNA hypomethylation underlies the change of histone acetylation status during decidualization in the promoter region of IGFBP-1. We previously showed that the change of histone acetylation status was associated with the induction of the IGFBP-1 by making the promoter regions accessible to transcription factors during decidualization [26]. Taken together, these observations suggest that, during decidualization, DNA hypomethylation of the promoter regions is maintained for the change of the histone modifications and the subsequent transcription factor binding.

Houshdaran et al. examined the DNA methylation statuses of the endometrium at the proliferative and mid-secretory phases by a genome-wide approach using 
Infinium HumanMethylation27K BeadChip [27]. They reported that 66 of 27,578 CpGs (0.2923\%) were differentially methylated between the two phases. There seems to be a discrepancy between their result and our result $(0.0048 \%)$. They used the cut-off of beta-values as $>0.136$ for extracting differentially methylated CpG sites, while we used the cut-off values as $>0.30$, which is generally accepted. Furthermore, other possible explanations for the discrepancy may lie in the different platforms of microarray, races, and study designs (in vivo and in vitro studies). Especially, since the samples in their in vivo study include other small structures such as capillaries and blood cells, the difference may have been overestimated.

By the comparison of DNA methylation pattern between the ESCs and other cell types, even the decidualized ESC-specific genes were already DNA hypomethylated in the non-decidualized ESCs, while other cell types showed DNA hypermethylation of the same genes. Furthermore, the ESC-specific DNA hypomethylation was linked to ESC-specific gene expression. The expressions of these genes only after decidualization should be caused by binding of appropriate transcription factors that would be coupled with histone acetylation and were important for the structural component of the decidualized endometrium. This suggests that the ESC-specific DNA hypomethylation profile defines the potential of differentiation in ESCs and contributes to decidualization. Taken together, ESCs are likely a terminally differentiated cell type, and decidualization would not be a differentiation process but simply a reaction to certain stimuli such as steroid hormones that result in morphological and gene expression changes. Notably, not all of the genes were specifically hypomethylated in the ESCs. However, since transcriptional repression of genes by DNA methylation is limited to specific genes with permanent silencing, it is not surprising that not all genes that were differentially expressed during decidualization showed ESC-specific DNA hypomethylation.

\section{Conclusion}

The present study showed that culturing ESCs with estrogen/progesterone does not distort the physiological pattern of DNA methylation, although histone modifications and mRNA expression dynamically change. In terms of the DNA methylation profile, ESCs before decidualization may have already been established as a fixed cell type. Taken our previous study on histone modifications into consideration [5], our results suggest that histone modifications rather than DNA methylation may have a preferential role in the regulation of gene expression during decidualization in ESCs, and that the regulation by histone modifications is supported by stable DNA hypomethylation.

\section{Methods}

\section{ESC isolation and cell culture}

ESCs were isolated and cultured as previously reported [5, 28, 29]. Human endometrial tissues were obtained from patients (aged 41-44 years) who underwent hysterectomy for myoma uteri or early stage of cervical cancer. They had a normal menstrual cycle. All endometrial samples were diagnosed as being in the late proliferative phase according to published criteria by histologic examination [30]. Tissue samples were washed with Phenol Red-free DMEM which contained $4 \mathrm{mM}$ glutamine, $50 \mu \mathrm{g} / \mathrm{mL}$ streptomycin, and $50 \mathrm{IU} / \mathrm{mL}$ penicillin, and minced into pieces of less than $1 \mathrm{~mm}^{3}$. ESCs were isolated as previously reported [31]. After the minced tissues underwent enzymatic digestion with $0.2 \%$ collagenase in a shaking water bath for $2 \mathrm{~h}$ at $37^{\circ} \mathrm{C}$, endometrial stromal cells were isolated by filtration through a $70-\mu \mathrm{m}$ nylon mesh. The filtrates were washed with the medium three times. The separated endometrial stromal cells were seeded at $10^{5}$ cells $/ \mathrm{cm}^{2}$ in $75-\mathrm{cm}^{2}$ tissue culture flasks and incubated in Phenol Red-free DMEM which contained glutamine, antibiotics, and 10\% dextran-coated charcoal-stripped fetal bovine serum at $37{ }^{\circ} \mathrm{C}, 95 \%$ air and 5\% CO2. At confluence, cells were resuspended with $1 \mathrm{x}$ trypsin-EDTA and subcultured into $75-\mathrm{cm}^{2}$ tissue culture flasks. When cells were harvested at $80 \%$ confluence after the first passage, ESCs were incubated with or without $\mathrm{E}\left(10^{-8} \mathrm{~mol} / \mathrm{L}\right)$ and MPA $\left(10^{-6}\right.$ $\mathrm{mol} / \mathrm{L}$ ) for 14 days at $37^{\circ} \mathrm{C}, 95 \%$ air, and $5 \% \mathrm{CO}_{2}$ to induce decidualization as previously reported [32]. The medium was changed every other day. Decidualization was confirmed by the induction of mRNA expression of $I G F B P-1$ and $P R L$, which are specific markers of decidualization, using the primers shown in Additional file 3: Table S3 [1, 29, 33]. The results were shown in Additional file 8: Figure S3. The ESCs were incubated and used for DNA methylation experiments.

Illumina Infinium HumanMethylation450 BeadChip assay Genomic DNA was isolated from the culture ESCs using a Qiagen Genomic DNA kit (Qiagen, Valencia, CA, USA). DNA methylation was analyzed with an Illumina Infinium assay with the HumanMethylation450 BeadChip (Illumina, San Diego, CA, USA), which interrogates a total of $482,421 \mathrm{CpGs}$ spread across the distal promoter regions of transcription start sites to 3 '-UTR of consensus coding sequences. Methylated and unmethylated signals were used to compute beta-values, which are quantitative scores of the DNA methylation levels, ranging from 0 (completely unmethylated) to 1 (completely methylated). The BeadChip was scanned on a BeadArray Reader (Illumina) according to the manufacturer's instructions. CpGs with "detection $p$ values" > 0.05 (computed from the background based on negative 
controls) and CpGs on Y chromosome were eliminated from further analysis, leaving 482,005 CpGs valid for use. CpGs whose methylation status differed between non-decidualized ESCs and decidualized ESCs were identified by the difference of beta-values. CpGs with beta values greater than 0.3 were extracted as differentially methylated CpGs. The raw data was deposited in the Gene Expression Omnibus (number GSE108143). We used NCBI Reference Sequence Database (https:// www.ncbi.nlm.nih.gov/refseq/) as reference genes.

\section{Differentially expressed genes between non-decidualized ESCs and decidualized ESCs}

Genome-wide mRNA expression data of two pairs of non-decidualized ESCs and decidualized ESCs were obtained from our previous RNA sequencing study [5]. RNA-seq raw data was deposited in the Gene Expression Omnibus (number GSE57010). Gene expression was mapped and quantified by TopHat [34], and cufflinks [35]. Gene expression values were calculated as fragments per kilobase of exon unit per million mapped reads (FPKM). The mean values in decidualized and non-decidualized ESCs were calculated, respectively. We defined the genes whose FPKM values increased or decreased more than 1.5-fold in decidualization as up-regulated or down-regulated genes during decidualization, respectively.

\section{Regions with different histone modification statuses between non-decidualized ESCs and decidualized ESCs}

The genome-wide statuses of the histone modifications of H3K4me3 and H3K27ac in decidualized and non-decidualized ESCs were obtained from our previous study of chromatin immuno-precipitation (ChIP) sequencing [5]. ChIP-seq raw data was deposited in the Gene Expression Omnibus (number GSE57010). Regions with different histone modifications were mapped and identified by Bowtie [36], followed by Model-based Analysis of ChIP-Seq (MACS) [37]. The regions with different histone modifications that were shared by the two individuals in the study were considered as the regions in which histone modification signals were altered by decidualization.

\section{Comparison of the DNA methylation status between ESCs and other cell types}

We obtained the DNA methylation data of 8 cell types other than ESCs examined by Illumina Infinium HumanMethylation450 BeadChip from gene expression omnibus database (human embryonic stem cells, brain and leukocyte from GSE52576; spleen, liver, omentum, pancreas and saliva from GSE 48472). Genes whose average beta-value from -300 to +700 bp in the ESCs was less than 0.2 and whose expression were increased or decreased more than 1.5 -fold during decidualization were extracted. Then, the average beta-values from -300 to $+700 \mathrm{bp}$ of the genes in each cell type were plotted in comparison with the ESCs. To investigate the genes that are specifically DNA hypomethylated in the ESCs, we extracted the genes that were DNA hypomethylated in the ESCs compared with any other cell types ( $\Delta$ beta-values $>0.3$ ).

\section{The expression statuses of the ESC-specific hypomethylated genes in other cell types}

The RNA sequencing of brain, liver, pancreas, spleen, saliva was retrieved from the GTEx portal (https://storage.googleapis.com/gtex_analysis_v7/rna_seq_data/GTEx_Analysis_ 2016-01-15_v7_RNASeQCv1.1.8_gene_reads.gct.gz). These expression data were scaled together with the expression data of the ESCs in R [38], and z-score was obtained. Of the ESC-specific hypomethylated and differentially expressed genes during decidualization (up-regulated 63 genes and down-regulated 43 genes), 51 and 35 genes were subjected to the analysis, respectively. The expression levels of these genes were compared between the ESCs (non-decidualized ESCs and decidualized ESC) and other cell types (brain, liver, pancreas, spleen and saliva). The expression statuses of these genes in each cell type were plotted.

\section{Sodium bisulfite sequencing}

Using the same DNA samples used in the BeadChip array, bisulfite reactions were performed using an EpiTect Bisulfite kit (Qiagen) with the following conditions: $95^{\circ} \mathrm{C}$ for $5 \mathrm{~min}, 65^{\circ} \mathrm{C}$ for $85 \mathrm{~min}, 95^{\circ} \mathrm{C}$ for 5 min, and $65^{\circ} \mathrm{C}$ for $175 \mathrm{~min}$ as previously reported $[14,39,40]$. The bisulfite converted DNA was amplified by PCR using the primer pairs shown in Additional file 3: Table S3 under the thermocycling conditions $\left(95^{\circ} \mathrm{C}\right.$ for $10 \mathrm{~min}$, and 40 cycles of $94{ }^{\circ} \mathrm{C}$ for $30 \mathrm{~s}, 57^{\circ} \mathrm{C}$ for $30 \mathrm{~s}$, and $72^{\circ} \mathrm{C}$ for $1 \mathrm{~min}$ followed by $10 \mathrm{~min}$ of final extension at $72{ }^{\circ} \mathrm{C}$ ). The resulting products were cloned into pGEM-T easy vector (Promega, Tokyo, Japan). After sequencing reaction using a BigDye Terminator V3.1 kit (Applied Biosystems, Foster City, CA, USA), sequencing was performed with a 3130xl Genetic Analyzer (Applied Biosystems) as previously reported [24, 41]. QUMA (http://quma. cdb.riken.jp/) was used to analyze the bisulfite sequencing data [42]. The bisulfite PCR primers are shown in Additional file 3: Table S3.

\section{Statistical analysis and bioinformatics}

The significance of difference between two groups was determined with an unpaired $t$ test. The significance of difference between multiple groups was determined with pairwise comparisons with $p$-value 
adjustment by Holm method. Statistical analyses were performed using SPSS for Windows version 11 (SPSS, Inc). Differences with $P<0.01$ or $P<0.05$ were considered significant. CpGs included in the upstream region, gene body region and downstream regions of genes, and the histone modified regions, were detected with Bedtools [43]. DAVID Bioinformatics Resources v. 6.7 was used to determine whether the functional annotation of the differentially expressed genes under ESC-specific DNA hypomethylation was enriched for specific GO terms [44]. In the GO analyses, $P<0.01$ were considered to indicate significant enrichment.

\section{Additional files}

Additional file 1: Table S1. Differentially expressed genes between the non-decidualized and decidualized ESCs and the number of the CpG sites around the transcription start sites ( -1500 to $4000 \mathrm{bp}$ ) of the genes. (XLSX $9 \mathrm{~kb}$ )

Additional file 2: Table S2. Regions with different histone modifications between the non-decidualized and decidualized ESCs. (XLSX $10 \mathrm{~kb}$ )

Additional file 3: Table S3. Primer pairs used in real-time RT-PCR and sodium bisulfite sequencing. (XLSX $10 \mathrm{~kb}$ )

Additional file 4: Table S4. ESC-hypomethylated genes (average betavalue from -300 to $+700 \mathrm{bp}$ is $<0.2$ ) and their methylation levels in other cell types. (XLSX $32504 \mathrm{~kb}$ )

Additional file 5: Table S5. Differentially expressed genes (106 genes) during decidualization under ESC-specific DNA hypomethylation. (XLSX $10 \mathrm{~kb})$

Additional file 6: Figure S1. DNA methylation status of the CpGs in UBE2D2 and COG5. A; DNA methylation statuses of the CpGs between + 4527 and +4784 of UBE2D2 were analyzed in the non-decidualized and decidualized ESCs. The CpG of +4527 was detected as differentially methylated CpG in Illumina HumanMethylation45K analysis. B; DNA methylation statuses of the CpGs between -433 to -194 of COG5 were analyzed in the non-decidualized and decidualized ESCs. The CpG of 379 was detected as differentially methylated CpG in Illumina HumanMethylation450K analysis. The diagrams show the distribution of CpGs. The position of the transcription start site is designated as +1 . Open and filled circles indicate unmethylated and methylated CpGs status, respectively. (JPG $379 \mathrm{~kb}$ )

Additional file 7: Figure S2. DNA methylation and histone modification statuses in the IGFBP-1 and PRL promoter regions. A and B; The DNA methylation statuses of CpGs in the promoter regions of IGFBP-1 (A) and PRL (B). A; Methylation status of all the CpGs between -501 and + 75 (27 CpGs) was analyzed in the non-decidualized and decidualized ESCs. B; DNA methylation status of all the CpGs between - 1966 and + 350 (11 CpGs) was analyzed in the non-decidualized and decidualized ESCs. The diagrams show the distribution of CpGs. The position of the transcription start site is designated as +1 . Open and filled circles indicate unmethylated and methylated CpGs status, respectively. C and D; H3K27ac and H3K4me3 statuses in the promoter regions of IGFBP-1 (C) and PRL (D). Peak call regions detected by the analysis using MACS are shaded. (JPG $392 \mathrm{~kb})$

Additional file 8: Figure S3. mRNA expression status of IGFBP-1 and PRL. mRNA expression of IGFBP-1 and PRL were analyzed by qRT-PCR using primers shown in Additional file: Table S3. GAPDH was used as an internal control. The value of mRNA was normalized to that of the internal control (GAPDH). (JPG $265 \mathrm{~kb}$ )

Additional file 9: Figure S4. Comparison of the expression status of the ESC-specific hypomethylated genes between the ESCs and other cell types. A and B; The mRNA expression statuses of ESC-specific hypomethylated and up-regulated (A) or down-regulated (B) genes. The expression levels of each gene in 7 cell types are indicated as dots (left column) and boxplots (right column). The significance of difference between multiple groups was determined with pairwise comparisons with $p$-value adjustment by Holm method. ESC, non-decidualized ESCs; dESC, decidualized ESCs. (JPG 788 kb)

\section{Abbreviations}

ChIP: chromatin immuno-precipitation; CO2: carbon dioxide;

COG5: component of oligomeric Golgi complex 5; CpG: Cytosine-phosphate-

Guanine; DAVID: Database for Annotation, Visualization and Integrated

Discovery; DMEM: Dulbecco's Modified Eagle's Medium;

DNA: Deoxyribonucleic acid; E: estrogen; EDTA: ethylenediaminetetraacetic acid; ESC: endometrial stromal cell; FPKM: fragments per kilobase of exon unit per million mapped reads; GO: gene ontology; H3K27ac: histone H3 lysine 27 acethylation; H3K4me3: histone H3 lysine 4 tri-methylation; IGFBP1: Insulin-like growth factor-binding protein 1; MACS: Model-based Analysis of ChIP-Seq; MPA: medroxyprogesterone acetate; mRNA: messenger ribonucleic acid; PCR: polymerase chain reaction; PRL: prolactin; QUMA: quantification tool for methylation analysis; SPSS: Statistical Package for Social Science; TFAP2A: transcription factor AP-2 alpha; UBE2D2: ubiquitin conjugating enzyme E2 D2; UTR: untranslated region

\section{Acknowledgements}

Authors thank anonymous reviewers.

\section{Funding}

This work was supported in part by JSPS KAKENHI Grants 16 K11142, 18 K09230, 18 K16772, 18 K09262, 18 K16801, 17 K11239 and 16 K11091 for Scientific Research from the Ministry of Education, Science, and Culture, Japan. The funders had no role in the study design, data collection and analysis, interpretation of data decision to publish, or preparation of the manuscript.

\section{Availability of data and materials}

The datasets generated and/or analyzed during the current study are available in the Gene Expression Omnibus (number GSE108143 for DNA methylation raw data; GSE57010 for RNA-seq raw data; GSE57010 for ChIP-seq raw data).

\section{Authors' contributions}

RM is the first author, and participated in the study designing, analysis and interpretation of the data and drafting of the manuscript. IT participated in analysis and interpretation of the data. MS, YM, SS, MO, TT and HT participated in the analysis of the data. NS participated in the study designing and drafting the manuscript. All authors have read and approved the final manuscript.

\section{Ethics approval and consent to participate}

This study was reviewed and approved by the Institutional Review Board of Yamaguchi University Graduate School of Medicine. Written informed consent to participate was obtained from the participants before the collection of any samples, and the specimens were irreversibly deidentified. All human tissues were handled in accordance with the Tenets of the Declaration of Helsinki.

\section{Consent for publication}

Written informed consent for publication was obtained from the participants.

\section{Competing interests}

The authors declare that they have no competing interests.

\section{Publisher's Note}

Springer Nature remains neutral with regard to jurisdictional claims in published maps and institutional affiliations.

Received: 4 February 2019 Accepted: 15 April 2019

Published online: 29 April 2019

\section{References}

1. Gellersen B, Brosens J. Cyclic AMP and progesterone receptor cross-talk in human endometrium: a decidualizing affair. J Endocrinol. 2003;178:357-72. 
2. Zhang QY. J.: update of Wnt signaling in implantation and decidualization. Reprod Med Biol. 2015;15:95-105.

3. Li B, Carey M, Workman JL. The role of chromatin during transcription. Cell. 2007;128:707-19.

4. Tamura I, Taketani T, Lee L, Kizuka F, Taniguchi K, Maekawa R, Asada H, Tamura H, Sugino N. Differential effects of progesterone on COX-2 and MnSOD expressions are associated with histone acetylation status of the promoter region in human endometrial stromal cells. J Clin Endocrinol Metab. 2011;96:E1073-82.

5. Tamura I, Ohkawa Y, Sato T, Suyama M, Jozaki K, Okada M, Lee L, Maekawa $\mathrm{R}$, Asada $\mathrm{H}$, Sato $\mathrm{S}$, et al. Genome-wide analysis of histone modifications in human endometrial stromal cells. Mol Endocrinol. 2014;28:1656-69.

6. Takizawa T, Nakashima K, Namihira M, Ochiai W, Uemura A, Yanagisawa M, Fujita N, Nakao M, Taga T. DNA methylation is a critical cell-intrinsic determinant of astrocyte differentiation in the fetal brain. Dev Cell. 2001;1: 749-58.

7. Kim M, Costello J. DNA methylation: an epigenetic mark of cellular memory. Exp Mol Med. 2017:49:e322.

8. Maier H, Colbert J, Fitzsimmons D, Clark DR, Hagman J. Activation of the early B-cell-specific mb-1 (Ig-alpha) gene by Pax-5 is dependent on an unmethylated Ets binding site. Mol Cell Biol. 2003;23:1946-60.

9. Hark AT, Schoenherr CJ, Katz DJ, Ingram RS, Levorse JM, Tilghman SM. CTCF mediates methylation-sensitive enhancer-blocking activity at the H19/lgf2 locus. Nature. 2000;405:486-9.

10. Bell AC, Felsenfeld G. Methylation of a CTCF-dependent boundary controls imprinted expression of the lgf2 gene. Nature. 2000;405:482-5.

11. Bird A. DNA methylation patterns and epigenetic memory. Genes Dev. 2002;16:6-21.

12. Wade PA. Methyl CpG-binding proteins and transcriptional repression. Bioessays. 2001;23:1131-7.

13. Sato S, Maeda C, Hattori N, Yagi S, Tanaka S, Shiota K. DNA methylationdependent modulator of Gsg2/Haspin gene expression. J Reprod Dev. 2011; 57:526-33.

14. Maekawa R, Sato S, Okada M, Lee L, Tamura I, Jozaki K, Kajimura T, Asada H, Yamagata Y, Tamura H, et al. Tissue-specific expression of estrogen receptor 1 is regulated by DNA methylation in a T-DMR. Mol Endocrinol. 2016;30: 335-47.

15. Ning X, Shi Z, Liu X, Zhang A, Han L, Jiang K, Kang C, Zhang Q. DNMT1 and EZH2 mediated methylation silences the microRNA-200b/a/429 gene and promotes tumor progression. Cancer Lett. 2015;359:198-205.

16. Wong CM, Wong CC, Ng YL, Au SL, Ko FC, Ng IO. Transcriptional repressive H3K9 and H3K27 methylations contribute to DNMT1-mediated DNA methylation recovery. PLoS One. 2011;6:e16702.

17. Bannister AJ, Kouzarides T. Regulation of chromatin by histone modifications. Cell Res. 2011:21:381-95.

18. Ball MP, Li JB, Gao Y, Lee JH, LeProust EM, Park IH, Xie B, Daley GQ, Church GM. Targeted and genome-scale strategies reveal gene-body methylation signatures in human cells. Nat Biotechnol. 2009;27:361-8.

19. Hellman A, Chess A. Gene body-specific methylation on the active $X$ chromosome. Science. 2007;315:1141-3.

20. Deaton AM, Webb S, Kerr AR, llingworth RS, Guy J, Andrews R, Bird A. Cell type-specific DNA methylation at intragenic $\mathrm{CpG}$ islands in the immune system. Genome Res. 2011;21:1074-86.

21. De Carvalho DD, You JS, Jones PA. DNA methylation and cellular reprogramming. Trends Cell Biol. 2010;20:609-17.

22. Liu H, Liu X, Zhang S, Lv J, Li S, Shang S, Jia S, Wei Y, Wang F, Su J, et al. Systematic identification and annotation of human methylation marks based on bisulfite sequencing methylomes reveals distinct roles of cell type-specific hypomethylation in the regulation of cell identity genes. Nucleic Acids Res. 2016;44:75-94.

23. Reik W. Stability and flexibility of epigenetic gene regulation in mammalian development. Nature. 2007:447:425-32.

24. Lee L, Asada H, Kizuka F, Tamura I, Maekawa R, Taketani T, Sato S, Yamagata $Y$, Tamura H, Sugino N. Changes in histone modification and DNA methylation of the StAR and Cyp19a1 promoter regions in granulosa cells undergoing luteinization during ovulation in rats. Endocrinology. 2013;154: 458-70.

25. Okada M, Lee L, Maekawa R, Sato S, Kajimura T, Shinagawa M, Tamura I, Taketani T, Asada H, Tamura H, Sugino N. Epigenetic changes of the Cyp11a1 promoter region in granulosa cells undergoing Luteinization during ovulation in female rats. Endocrinology. 2016;157:3344-54.
26. Tamura I, Asada H, Maekawa R, Tanabe M, Lee L, Taketani T, Yamagata Y, Tamura H, Sugino N. Induction of IGFBP-1 expression by CAMP is associated with histone acetylation status of the promoter region in human endometrial stromal cells. Endocrinology. 2012;153:5612-21.

27. Houshdaran S, Zelenko Z, Irwin JC, Giudice LC. Human endometrial DNA methylome is cycle-dependent and is associated with gene expression regulation. Mol Endocrinol. 2014;28:1118-35.

28. Matsuoka A, Kizuka F, Lee L, Tamura I, Taniguchi K, Asada H, Taketani T, Tamura H, Sugino N. Progesterone increases manganese superoxide dismutase expression via a CAMP-dependent signaling mediated by noncanonical Wnt5a pathway in human endometrial stromal cells. J Clin Endocrinol Metab. 2010;95:E291-9.

29. Tamura I, Shirafuta Y, Jozaki K, Kajimura T, Shinagawa M, Maekawa R, Taketani T, Asada H, Sato S, Tamura H, Sugino N. Novel function of a transcription factor WT1 in regulating Decidualization in human endometrial stromal cells and its molecular mechanism. Endocrinology. 2017;158:3696-707.

30. Noyes RW, Hertig AT, Rock J. Dating the endometrial biopsy. Am J Obstet Gynecol. 1975:122:262-3.

31. Sugino N, Karube-Harada A, Sakata A, Takiguchi S, Kato H. Nuclear factorkappa $B$ is required for tumor necrosis factor-alpha-induced manganese superoxide dismutase expression in human endometrial stromal cells. J Clin Endocrinol Metab. 2002;87:3845-50.

32. Sugino N, Kashida S, Takiguchi S, Nakamura Y, Kato H. Induction of superoxide dismutase by decidualization in human endometrial stromal cells. Mol Hum Reprod. 2000;6:178-84.

33. Tamura I, Sato S, Okada M, Tanabe M, Lee L, Maekawa R, Asada H, Yamagata Y, Tamura H, Sugino N. Importance of C/EBPbeta binding and histone acetylation status in the promoter regions for induction of IGFBP-1, PRL, and Mn-SOD by CAMP in human endometrial stromal cells. Endocrinology. 2014;155:275-86.

34. Trapnell C, Pachter L, Salzberg SL. TopHat: discovering splice junctions with RNA-Seq. Bioinformatics. 2009;25:1105-11.

35. Trapnell C, Williams BA, Pertea G, Mortazavi A, Kwan G, van Baren MJ, Salzberg SL, Wold BJ, Pachter L. Transcript assembly and quantification by RNA-Seq reveals unannotated transcripts and isoform switching during cell differentiation. Nat Biotechnol. 2010:28:511-5.

36. Langmead B, Trapnell C, Pop M, Salzberg SL. Ultrafast and memory-efficient alignment of short DNA sequences to the human genome. Genome Biol. 2009;10:R25.

37. Zhang Y, Liu T, Meyer CA, Eeckhoute J, Johnson DS, Bernstein BE, Nusbaum C, Myers RM, Brown M, Li W, Liu XS. Model-based analysis of ChIP-Seq (MACS). Genome Biol. 2008;9:R137.

38. Gentleman RC, V.; Huber, W.; Hahne F: Genefilter: methods for filtering genes from high-throughput experiments. R package version 1.64.0., 1.64.0. edition; 2018.

39. Sato S, Maekawa R, Yamagata Y, Asada H, Tamura I, Lee L, Okada M, Tamura $\mathrm{H}$, Sugino N. Potential mechanisms of aberrant DNA hypomethylation on the $x$ chromosome in uterine leiomyomas. J Reprod Dev. 2014;60:47-54.

40. Yamagata Y, Nishino K, Takaki E, Sato S, Maekawa R, Nakai A, Sugino N. Genome-wide DNA methylation profiling in cultured eutopic and ectopic endometrial stromal cells. PLoS One. 2014:9:e83612.

41. Maekawa R, Sato S, Yamagata Y, Asada H, Tamura I, Lee L, Okada M, Tamura H, Takaki E, Nakai A, Sugino N. Genome-wide DNA methylation analysis reveals a potential mechanism for the pathogenesis and development of uterine leiomyomas. PLoS One. 2013;8:e66632.

42. Kumaki Y, Oda M, Okano M. QUMA: quantification tool for methylation analysis. Nucleic Acids Res. 2008;36:W170-5.

43. Quinlan AR, Hall IM. BEDTools: a flexible suite of utilities for comparing genomic features. Bioinformatics. 2010;26:841-2.

44. Huang d W, Sherman BT, Tan Q, Kir J, Liu D, Bryant D, Guo Y, Stephens R, Baseler MW, Lane HC, Lempicki RA. DAVID bioinformatics resources: expanded annotation database and novel algorithms to better extract biology from large gene lists. Nucleic Acids Res. 2007;35:W169-75. 